\title{
Rationale Tumordokumentation und Qualitäts- sicherung zur Verbesserung der Versorgungsqualität für Lungenkrebspatienten
}

\author{
Rational Tumour Documentation and Quality Assurance to Improve the \\ Standard of Medical Care for Lung Cancer Patients
}

Autoren

Institut
T. Blum, N. Schönfeld, J. Kollmeier, W. Ammenwerth, S. Delis, W. Grüning, W. Nehls, S. Thiel, T. T. Bauer ${ }^{1}$

Klinik für Pneumologie, Lungenklinik Heckeshorn, HELIOS Klinikum Emil von Behring, Berlin (Leiter: Prof. Dr. Torsten T. Bauer)

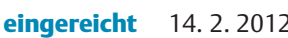
akzeptiert nach Revision 25. 2. 2012

Bibliografie

Dol http://dx.doi.org/

$10.1055 / \mathrm{s}-0032-1308902$

Pneumologie 2012; 66: 224-230

(c) Georg Thieme Verlag KG

Stuttgart · New York

ISSN 0934-8387

Korrespondenzadresse Dr. med. Torsten Blum, M.B.A.

Klinik für Pneumologie Lungenklinik Heckeshorn HELIOS Klinikum Emil von Behring Walterhöferstr. 11

14165 Berlin

torsten-gerriet.blum@helioskliniken.de

\section{Zusammenfassung}

$\nabla$

Die Datenlage zur Versorgungsqualität von Patienten mit Lungenkrebs in Deutschland ist unzureichend. Zwar existiert mit der S3-Leitlinie Lungenkarzinom aus dem Jahr 2010 eine gute wissenschaftliche Handlungsgrundlage für die mitunter komplexen Behandlungspfade, jedoch ist nicht belegt, inwieweit die entsprechenden Leitlinienempfehlungen bundesweit umgesetzt werden oder welche Therapien überhaupt bei einer der prognostisch schlechtesten Tumorentitäten zur Anwendung kommen. Im Rahmen des Nationalen Krebsplans aus dem Jahr 2008 wurden konkrete Handlungsziele zur systematischen Verbesserung der onkologischen Versorgung in Deutschland formuliert. Als ein wesentliches Ziel wird eine bundesweite Neugestaltung und Vereinheitlichung von Tumordokumentation und Qualitätssicherung zur nachhaltigen Steigerung der Versorgungsqualität gefordert. Dieser Übersichtsartikel erläutert die zentralen Begrifflichkeiten und untersucht, inwieweit die Handlungsziele des Nationalen Krebsplanes beim Lungenkarzinom bislang umgesetzt wurden.

\section{Einleitung}

Die Behandlung von Lungenkrebskranken ist ein komplexer und im Einzelfall sehr variabler Prozess. Die S3-Leitlinie Lungenkarzinom von 2010 bildet die hierzulande gültige, systematische, evidenz- und konsensbasierte Handlungsempfehlung [1]. Zu dem Grad der Verbreitung und Implementierung der S3-Leitlinie Lungenkarzinom in Deutschland existieren aber zurzeit keine systematischen Untersuchungen. Zahlreiche Fachdisziplinen unterschiedlicher sektoraler Zugehörigkeiten sowie nicht-ärztliche Professionen sind in Abhängigkeit von den individuellen Krankheitsverläufen in den Prozess der Lungenkrebsversorgung involviert. Zwar liegt der Behandlungs-

\section{Abstract \\ $\nabla$}

The data on the quality of care of patients with lung cancer in Germany are insufficient. Although the National Lung Cancer Guideline from 2010 provides a good scientific basis for the management of the frequently complex pathways, no evidence exists showing how the relevant guideline recommendations are implemented nationwide or which treatment options generally are chosen in a tumour entity with one of the poorest prognoses. As part of the National Cancer Plan 2008, specific targets have been formulated for the systematic improvement of cancer care in Germany. As a main goal, the national re-organisation and harmonisation of tumor documentation and quality assurance are required for a sustainable improvement in the quality of care. This review article first describes the relevant terms and then examines how the specific targets of the National Cancer Plan have been implemented so far with regard to lung cancer care.

schwerpunkt in Lungenkliniken bzw. entsprechend spezialisierten stationären Abteilungen, dennoch ist die publizierte Datenlage zur Versorgung von Lungenkrebspatienten in Deutschland insgesamt unzureichend und erlaubt insbesondere keine umfassenden Rückschlüsse auf die regionale bzw. nationale Versorgungsqualität [2]. Es ergibt sich somit bei der in Deutschland dritthäufigsten Krebsneuerkrankung mit der höchsten Zahl der Sterbefälle [3] eine Diskrepanz zwischen der Tatsache, dass wir zwar auf gute wissenschaftliche Handlungsempfehlungen zurückgrei-

\footnotetext{
* Die Erstellung, Pflege und Auswertung der Tumordatenbank der Lungenklinik Heckeshorn wurde durch die Stiftung Oskar-Helene-Heim, Berlin, unterstützt.
} 
Handlungsfeld 1: Weiterentwicklung der Krebsfrüherkennung

Ziel 1: Die informierte Inanspruchnahme der im Nutzen belegten Krebsfrüherkennungsprogramme der gesetzlichen Krankenkassen wird gesteigert.

Ziel 2: Die Krebsfrüherkennungsuntersuchungen, die nachweislich in der Lage sind, die Mortalität an der Zielerkrankung zu senken, berücksichtigen die europäischen Empfehlungen an systematische populationsbasierte Screeningprogramme.

Ziel 3: Die Krebsfrüherkennungsprogramme werden hinsichtlich ihres Nutzens (v.a. Mortalitätssenkung) unter Einbindung der epidemiologischen Landeskrebsregister evaluiert.

\section{Handlungsfeld 3: Sicherstellung einer effizienten onkologischen} Behandlung

Ziel 10: Alle Patienten erhalten einen fairen und schnellen Zugang zu nachweislich wirksamen innovativen Krebstherapien.

Abb. 1 Zielmatrix des Nationalen Krebsplans (adaptiert nach [5]).

fen können, aber die tatsächliche Versorgungsqualität der Patienten nahezu unbekannt ist. Das von Ollenschläger bereits 2001 skizzierte Spannungsfeld „zwischen steigenden Kosten infolge erhöhter Nachfrage nach Gesundheitsdienstleistungen, immer teurer werdenden Technologien, alternden Bevölkerungen, Qualitätsschwankungen mit zum Teil inadäquater Gesundheitsversorgung (Über- und Unterversorgung) und dem selbstverständlichen Wunsch der Leistungsanbieter bzw. der Patienten nach bestmöglicher Versorgung“ [4] ist eine Verpflichtung, diese Fragestellungen beim Lungenkarzinom systematisch wissenschaftlich zu untersuchen.

Mit dem Nationalen Krebsplan wurde schon 2008 unter Beteiligung relevanter Interessengruppen eine übergeordnete Zielmatrix für Deutschland postuliert ( $\bullet$ Abb.1) [5]. Mittlerweile wurden eine systematische Bestandsaufnahme und detaillierte Problemerfassung durchgeführt und daraus Handlungsempfehlungen abgeleitet [6].

Dieser Übersichtsartikel widmet sich vor dem Hintergrund der aktuellen Thematik zwei etablierten Verfahren, der Tumordokumentation und der Qualitätssicherung, welche beide methodisch geeignet sind, die Versorgungsqualität beim Lungenkarzinom umfassend abzubilden. Als zwei Seiten einer Medaille sollen diese beiden Verfahren nach einer allgemeinen Betrachtung dahingehend näher beleuchtet werden, wie sie, sinnvoll kombiniert und rational eingesetzt, beim Lungenkarzinom spezifisch Anwendung finden können.
Handlungsfeld 2: Weiterentwicklung der onkologischen Versorgungsstrukturen und der Qualitätssicherung

Ziel 4: Alle Krebspatienten erhalten eine qualitativ hochwertige Versorgung, unabhängig von Alter, Geschlecht, Herkunft, Wohnort und Versichertenstatus.

Ziel 5: Es existieren einheitliche Konzepte und Bezeichnungen für die Qualitätssicherung und Qualitätsförderung und Zertifizierung onkologischer Behandlungseinrichtungen.

Ziel 6: Für alle häufigen Tumorarten existieren evidenzbasierte Behandlungsleitlinien der höchsten methodischen Entwicklungsstufe (sog. S3-Leitlinien), die von den onkologischen Behandlungseinrichtungen umgesetzt werden.

Ziel 7: Eine sektorenübergreifende, integrierte onkologische Versorgung ist gewährleistet.

Ziel 8: Es existiert eine aussagekräftige onkologische Qualitätsberichterstattung für Leistungserbringer, Entscheidungsträger und Patienten.

Ziel 9: Alle Krebspatienten erhalten bei Bedarf eine angemessene psychoonkologische Versorgung.

\section{Handlungsfeld 4: Stärkung der Patientenorientierung}

Ziele 11a und 11b: Es liegen für alle Krebspatienten und ihre Angehörigen niederschwellige, zielgruppenorientierte und qualitätsgesicherte Informationsangebote (Ziel 11a) und qualitätsgesicherte Beratungs- und Hilfsangebote (Ziel 11b) vor.

Ziel 12a: Alle in der onkologischen Versorgung tätigen Leistungserbringer verfügen über die notwendigen kommunikativen Fähigkeiten zu einem adäquaten Umgang mit Krebspatienten und ihren Angehörigen.

Ziel 12b: Stärkung der Patientenkompetenz.

Ziel 13: Die Patientinnen und Patienten werden aktiv in die Entscheidung über medizinische Maßnahmen einbezogen.

\section{Definitionen und Begriffserläuterungen}

„Alle reden von Qualität und Qualitätsmanagement - aber meinen wir das Gleiche?“ Mit dieser provokativen Frage leitet das Glossar der Arbeitsgruppe „Qualitätsmanagement in der Medizin“ der Deutschen Gesellschaft für Medizinische Informatik, Biometrie und Epidemiologie (GMDS) ein [7]. Angesichts unterschiedlicher Anspruchsgruppen mit z.T. differierenden Interessenlagen im Bereich der Lungenkrebsversorgung [8] sollen daher zunächst die Begriffe Qualität, Qualitätssicherung, Tumordokumentation definiert und weitergehend erläutert werden.

\section{Qualität}

In der Literatur finden sich zahlreiche Definitionen von Qualität, welche im GMDS-Glossar umfassend gelistet und kommentiert wurden. Speziell für den Gesundheitsbereich haben zwei Definitionen von Qualität Anerkennung und Verwendung gefunden [7]:

- "Quality of care is the extent to which actual care is in conformity with preset criteria for good care." Diese Definition von Donabedian enthält den Übereinstimmungsgrad der medizinischen Dienstleistung mit den zuvor definierten Anforderungen. Donabedian formulierte zudem in seiner Pionierarbeit für den Gesundheitssektor die auch heute noch gebräuchliche Unterteilung von Qualität in Struktur-, Prozessund Ergebnisqualität [9]. 
- „Qualität im Gesundheitswesen bedeutet eine ausreichende und zweckmäßige, d.h. patienten- und bedarfsgerechte, an der Lebensqualität orientierte, fachlich qualifizierte, aber auch wirtschaftliche medizinische Versorgung mit dem Ziel, die Wahrscheinlichkeit erwünschter Behandlungsergebnisse bei Individuen und in der Gesamtbevölkerung zu erhöhen." Diese neuere, konkretere Definition von Ruprecht bezieht die Anforderung der sozialen Gesetzgebung sowie der ärztlichen Berufsordnung ein [10].

\section{Qualitätssicherung}

Qualitätssicherung hielt ursprünglich in den 60er-Jahren in Prozesse der industriellen Produktion im Rahmen einer angestrebten Qualitätsverbesserung Einzug, damit sich Unternehmen von der Konkurrenz abheben und dadurch eigene Absatzchancen von Produkten bei zunehmend gesättigten Märkten steigern konnten. Die DIN-EN-ISO-9000-Norm definiert Qualitätssicherung als „Teil des Qualitätsmanagements, der auf das Erzeugen von Vertrauen darauf gerichtet ist, dass Qualitätsanforderungen erfüllt werden [11]“. Qualitätssicherung hat in der Folge im Dienstleistungssektor und hierüber auch im Gesundheitswesen Fuß gefasst. Das Grundprinzip der Qualitätssicherung beruht auf der systematischen Dokumentation und Analyse qualitätsrelevanter Daten, um in der retrospektiven Betrachtung Rückschlüsse auf zukünftige Optimierungspotenziale der eigenen Leistungserbringung zu ziehen. Darüber hinaus erlaubt Qualitätssicherung, eigene Strukturen, Prozesse und Ergebnisse nach innen und außen transparent und somit nachprüfbar zu machen, und ferner den Vergleich korrespondierender Qualitätskategorien zwischen verschiedenen Leistungserbringern. In Abhängigkeit davon, ob Qualitätssicherung durch den Leistungserbringer selbst oder durch externe Institutionen veranlasst wird, findet die Unterscheidung zwischen interner und externer Qualitätssicherung Anwendung. Der Gesetzgeber hat die Verpflichtung medizinischer Leistungserbringer zur Qualitätssicherung in den Sozialgesetzbüchern V und XI verankert [7].

\section{Tumordokumentation}

Die Tumordokumentation wird von deutschen Institutionen nicht explizit definiert, meint aber nach gängiger Auffassung die systematische Datenerfassung bei Tumorerkrankungen. Die übergeordneten Ziele von Tumordokumentation sind:

- Epidemiologie von Tumorerkrankungen

- Qualitätssicherung und kontinuierliche Verbesserung der onkologischen Versorgung

- Tumorforschung

In Deutschland hat sich mit der Ausbildung von epidemiologischen und klinischen Krebsregistern eine Doppelstruktur der systematischen Tumordokumentation mit unterschiedlichen Zielsetzungen entwickelt.

\section{Epidemiologische Krebsregister}

Das 1926 gegründete Hamburgische Krebsregister gilt als weltweit erster Prototyp eines epidemiologischen Krebsregisters [12]. Heutzutage fokussieren epidemiologische Krebsregister bei ihrer Tumordokumentation auf folgende Schwerpunkte [13]:

- Gesundheitsberichterstattung von epidemiologischen Krebsdaten (u.a. Inzidenz, Prävalenz, Mortalität) in Quer- und Längsschnittanalysen bzw. im regionalen Vergleich

- epidemiologische Forschung

- Planung von Prävention und Früherkennung
Um diesen Aufgaben gerecht zu werden, ist eine vollständige Erfassung aller Krebserkrankungsfälle in der zugehörigen Region eines epidemiologischen Krebsregisters eine Voraussetzung [14]. Die aktuell bestehenden Strukturen, aber auch Probleme der epidemiologischen Tumordokumentation lassen sich durch die Historie Deutschlands mit vorübergehender Teilung und dem nachfolgenden Ausbau föderaler Strukturen erklären. In der DDR war das Nationale Krebsregister der Wegbereiter für das 1993 neu formierte Gemeinsame Krebsregister (GKR) der Bundesländer Berlin, Brandenburg, Mecklenburg-Vorpommern, Sachsen-Anhalt, Sachsen und Thüringen. In der alten Bundesrepublik wurde 1967 (erstmals in einem Flächenstaat) das Epidemiologische Krebsregister Saarland gegründet. Das Gesetz über Krebsregister (KRG), das von 1995 bis 1999 Gültigkeit besaß, verpflichtete alle Bundesländer, eigene epidemiologische Krebsregister zu installieren. Ausnahmeregelungen führten jedoch zunächst zu Parallelentwicklungen in Bundesländern mit inkongruenten Systemen. Folgerichtig konstituierte sich 2004 die Gesellschaft der epidemiologischen Krebsregister in Deutschland (GEKID) mit der vorrangigen Zielsetzung, bestehende Ungleichheiten zu harmonisieren. Ein weiterer Schritt war die Einführung des Bundeskrebsregisterdatengesetzes (BKRG) 2009, das von den epidemiologischen Krebsregistern der Bundesländer eine Zusammenführung ihrer anonymisierten Daten zu einem einheitlichen Datensatz für Deutschland einfordert. Für die statistischen Analysen auf Bundesebene autorisierte das BKRG das Zentrum für Krebsregisterdaten (ZfKD) am Robert-Koch-Institut (RKI) als Nachfolger der Dachdokumentation Krebs am RKI [12,13,15-18].

Im Hinblick auf die Vollständigkeit der einzelnen epidemiologischen Krebsregister, d.h. die Erfassung aller ärztlich diagnostizierten Krebserkrankungen der Einwohner im Einzugsgebiet des Krebsregisters, konnten ZfKD und GEKID zuletzt einen positiven Trend verzeichnen. In der aktuellen Berichterstattung 2010 wird eine vollzählige Erfassung in sieben Bundesländern (Bayern, Bremen, Hamburg, Niedersachsen, Saarland, Sachsen, SchleswigHolstein) genannt, legt man den international hierfür etablierten Cut-off von $90 \%$ und mehr für die Erfassungsquote bei beiden Geschlechtern zugrunde. In drei Bundesländern (Baden-Württemberg, Hessen, Nordrhein-Westfalen) lagen keine flächendeckenden Daten vor [3].

\section{Klinische Krebsregister}

„Ein klinisches Krebsregister strebt die Erfassung aller Krebspatientinnen und Krebspatienten an, die an einer bestimmten Einrichtung bzw. einem Einrichtungsverbund oder (idealerweise) in einem definierten Einzugsgebiet behandelt wurden. Hauptziele dieser Erfassung sind die Qualitätssicherung und die Darstellung der Ergebnisqualität der gesamten Behandlung von Krebspatienten." So lautet die Definition des Kooperationsverbunds Qualitätssicherung durch Klinische Krebsregister (KoQK), einem Zusammenschluss von Personen aus Politik, Selbstverwaltung, Wissenschaft, Tumorzentren und anderen für Krebskranke engagierten Institutionen [19]. Klinische Krebsregister versuchen daher, komplette Behandlungsketten von Krebspatienten abzubilden, angefangen von Diagnostik über standardisierte Therapiemodalitäten bis hin zu Nachsorgemaßnahmen. Dabei macht klinische Tumordokumentation idealerweise nicht vor Sektorengrenzen halt, sondern erfasst die ambulante, stationäre und rehabilitative Versorgung von Tumorpatienten gleichermaßen. Nachfolgend sind die wesentlichen Zielsetzungen klinischer Krebsregister gelistet $[14,19,20]$ : 
- Darstellung und Qualitätssicherung onkologischer Versorgung

- Strukturqualität

- Prozessqualität (z. B. Befolgen der Empfehlungen von interdisziplinären Tumorkonferenzen oder Leitlinien)

- Ergebnisqualität (z. B. Überlebenszeiten verschiedener Therapieformen im Vergleich)

- Benchmarking von unterschiedlichen Leistungserbringern bei Teilaspekten der onkologischen Versorgung

- Rückmeldung der aggregierten klinischen Registerdaten an Leistungserbringer mit dem Ziel der kontinuierlichen Verbesserung der onkologischen Versorgung (z. B. strukturierter Dialog im Rahmen von Qualitätskonferenzen)

- Kooperation mit epidemiologischen Krebsregistern (bidirektionaler Datenfluss)

- klinische und Versorgungsforschung

Klinische Krebsregister in Deutschland sind - sieht man von isolierter onkologischer Dokumentation einzelner Leistungserbringer ab - traditionell an Tumorzentren gekoppelt. Gemäß der Definition der Arbeitsgemeinschaft Deutscher Tumorzentren (ADT) ist ein Tumorzentrum „das organisatorische Dach, unter dem interdisziplinär und sektoral koordinierte Aktivitäten gebündelt sind. Ein Tumorzentrum vernetzt stationäre Einrichtungen aller Versorgungsstufen einschließlich Comprehensive Cancer Center und organspezifischer interdisziplinärer Zentren und ambulante Einrichtungen und Leistungserbringer in einer definierten Region. Es führt ein klinisches Krebsregister." Aktuell haben sich deutschlandweit 49 Tumorzentren in der ADT zusammengeschlossen [21].

\section{Der Umsetzungsstand des Nationalen Krebsplan beim Lungenkarzinom \\ $\nabla$}

Die Weiterentwicklung onkologischer Versorgungsstrukturen und deren Qualitätssicherung ist im Nationalen Krebsplan Deutschlands als eines von insgesamt vier Handlungsfeldern verankert ( $\bullet$ Abb. 1). Der gemeinsame Masterplan des Bundesministeriums für Gesundheit (BMG), der Deutschen Krebsgesellschaft (DKG), der Deutschen Krebshilfe und der ADT formulierte 2008 für dieses Handlungsfeld sechs übergeordnete Ziele, u. a. die Forderungen nach Erstellung und Umsetzung von S3-Behandlungsleitlinien für alle häufigen Tumorarten, einheitlicher Qualitätssicherung und Zertifizierung onkologischer Behandlungseinrichtungen sowie aussagekräftiger onkologischer Qualitätsberichterstattung [5]. Laut dem aktuellen Informationspapier des BMG vom Januar 2012 befinden sich die genannten Teilprojekte des Nationalen Krebsplans noch in Planungs- bzw. erst frühen Durchführungsstadien. Das Zwischenresümee ist aber mit Ausnahme des Lungenkarzinoms positiv ausfallen, ist es doch gelungen, einerseits alle relevanten Interessengruppen für die anderen ausgesuchten Tumorentitäten einzubinden und andererseits den Istzustand mit seinen z.T. hochkomplexen Problemen detailliert zu beschreiben und daraus klar definierte Handlungsempfehlungen abzuleiten [6]. Für den Lungenkrebs wurde jedoch nichts erreicht, insbesondere nicht im Hinblick auf den zentralen Vorschlag für politisches Handeln, der im Vorfeld der Erstellung des Nationalen Krebsplans gemacht wurde, die Tabakprävention [22].

Mit der Veröffentlichung der S3-Leitlinie zum Lungenkarzinom 2010 wurde ein Teilziel realisiert, auch wenn bis dato keine systematischen Untersuchungen im Hinblick auf deren Umsetzung auf regionaler oder nationaler Ebene publiziert wurden [1]. Eine
Tab. 1 Handlungsempfehlungen der „Aufwand-Nutzen-Abschätzung zum Ausbau und Betrieb bundesweit flächendeckender klinischer Krebsregister“ durch die Fa. Prognos [28].

\begin{tabular}{|l|l|}
\hline Gesetzliche Verbindlichkeit herstellen \\
\hline Aufgabenprofil gemeinsam mit Datennutzern definieren \\
\hline Standards definieren \\
\hline Datenschutzrechtliche Vorgaben vereinheitlichen \\
\hline Regionale Einzugsgebiete definieren \\
\hline Dezentrale und zentrale Dokumentationsstrukturen etablieren \\
\hline Neutralität und Unabhängigkeit der Register sicherstellen \\
\hline Meldeweg über die klinischen Krebsregister festschreiben \\
\hline Follow-Up-Ermittlung erleichtern \\
\hline Meldeaufwand vereinfachen \\
\hline Regelmäßige Rückmeldungen etablieren \\
\hline Zur Kooperation mit Organkrebszentren verpflichten \\
\hline Meldevergütungen harmonisieren \\
\hline Doppeldokumentationsstrukturen abbauen \\
\hline Finanzierung \\
\hline
\end{tabular}

einheitliche Qualitätssicherung und Zertifizierung onkologischer Einrichtungen zur Lungenkrebsbehandlung ist hingegen nicht absehbar, stattdessen konkurrieren etablierte Verfahren verschiedener medizinischer Fachgesellschaften bzw. der Kostenträger/Kassenärztlichen Vereinigungen miteinander [23-26]. Im Hinblick auf eine valide nationale Qualitätsberichterstattung für das Lungenkarzinom existieren zwar relativ robuste epidemiologische Zahlen durch ZfKD und GEKID [3], jedoch keine flächendeckenden Auswertungen klinischer Krebsregistrierung auf Bundesebene. Unter Federführung des Tumorzentrums Berlin präsentierte die ADT anlässlich der 3.Bundesweiten Onkologischen Qualitätskonferenz des KoQK während des 29. Deutschen Krebskongresses in Berlin 2010 immerhin Ergebniszahlen von 28 klinischen Krebsregistern zum Lungenkarzinom. In dieser retrospektiven Beobachtungsstudie wurden in dem Diagnosezeitraum von 1998 bis 2005 rund 30\% aller prognostizierten neu diagnostizierten Lungenkarzinomfälle in Deutschland erfasst [27]. Der Nationale Krebsplan schreibt den klinischen Krebsregistern eine Schlüsselstellung in der Neugestaltung der onkologischen Versorgungslandschaft $\mathrm{zu}$, indem sie durch eine sektorenübergreifende Dokumentation der onkologischen Behandlungsabläufe eine transparente Darstellung und Berichterstattung onkologischer Versorgungsqualität sowie damit letztendlich auch eine Steuerung der zugrunde liegenden Versorgungsstrukturen ermöglichen [5]. Das BMG gab hierzu ein Gutachten zur „Aufwand-Nutzen-Abschätzung zum Ausbau und Betrieb bundesweit flächendeckender klinischer Krebsregister" in Auftrag, das im Oktober 2010 veröffentlicht wurde [28]. Das Gutachten spricht sich klar für den Ausbau einer bundesweit flächendeckenden Tumordokumentation durch klinische Krebsregister mit dem Ziel einer gebündelten onkologischen Qualitätssicherung aus, sieht aber bundesweit hierzu noch einen erheblichen Handlungsbedarf. Als Positivbeispiele werden die klinischen Krebsregister Bayerns und Brandenburgs genannt. Die laufenden jährlichen Erhaltungskosten für eine flächendeckende klinische Tumordokumentation werden in dem Gutachten in Abhängigkeit vom Einzugsgebiet mit $0,63 €$ bis $0,81 €$ pro Einwohner prognostiziert. - Tab. 1 nennt die in dem Gutachten aufgestellten Handlungsempfehlungen als Grundvoraussetzungen einer bundesweit funktionierenden klinischen Tumordokumentationsstruktur, von denen einzelne Punkte in den nächsten Abschnitten mit Fokussierung auf das Lungenkarzinom detaillierter betrachtet werden sollen [28]. 


\section{Wege zur rationalen Tumordokumentation und Qualitätssicherung}

\section{Abbau von Doppel- und Mehrfachdokumentations- strukturen}

Eine Datenökonomie (Mehrfachnutzung von Daten) und Datensparsamkeit (Erfassung ausschließlich relevanter Daten) muss angesichts knapper personeller Ressourcen im Gesundheitswesen höchste Priorität besitzen. Jeder erfasste Parameter sollte einen Nutzen besitzen, und dieser Nutzen sollte den entsprechenden Dokumentationsaufwand für einen Parameter überwiegen. Eine von der Lenkungsgruppe des Nationalen Krebsplans beauftragte AG Dokumentation stellte in einem Arbeitspapier am Beispiel des Mammakarzinoms aktuell bestehende, ressourcenverbrauchende Fehlentwicklungen bzw. Parallelstrukturen sowie Lösungswege zu deren Abbau dar. Hiernach überschreiten Dokumentationsanforderungen oft bei weitem das eigentliche Maß, das tatsächlich für Transparenz, Organisation und Optimierung selbst komplexer onkologischer Versorgung notwendig wäre. Redundanz in den unterschiedlichen Dokumentationsanforderungen wird als wesentliches Problem angeführt und mit einer Untersuchung untermauert, wonach beim Mammakarzinom rund zwei Drittel der Parameter zwei- bis vierfach erfasst werden müssen. Zumeist stehen nur divergente Datenerfassungs- und Meldewege für die jeweiligen Dokumentationssysteme zur Verfügung. Schnittstellenlösungen zwischen den verschiedenen Erfassungssystemen fehlen oft. Mitunter liegen unterschiedliche Wertedefinitionen bei gleichen Parametern vor [29]. Die derzeitige Situation bei der Tumordokumentation des Lungenkarzinoms unterscheidet sich nicht hinsichtlich der genannten Probleme. Exemplarisch sei auf die verschiedenen Dokumentationsanforderungen beim Lungenkarzinom verwiesen, die erhebliche Redundanzen aufweisen ( $\bullet$ Tab.2).

\section{Definition von Dokumentationsstandards}

Bei der Definition von Standards im Hinblick auf Mindestinhalte und Kompatibilität der Datenstrukturen von Krebsregistern hat der KoQK 2008 in Zusammenarbeit mit den epidemiologischen Krebsregistern und der DKG den einheitlichen onkologischen Basisdatensatz für alle Krebsarten legitimiert, der bundesweit Mehrfachdokumentationen bei epidemiologischer und klinischer Tumordokumentation, aber auch bei der Qualitätssicherung im Rahmen der Zertifizierung von Organkrebszentren bzw. der Evaluation bzgl. der Adhärenz von Leitlinienempfehlungen verhindern soll. Der einheitliche onkologische Basisdatensatz ist modular aufgebaut ( Tab.3) und kann von den gängigen elek-
Tab. 2 Anforderungen an Tumordokumentation und Qualitätssicherung beim Lungenkarzinom.

\begin{tabular}{|l|}
\hline Tumordokumentation \\
\hline interne klinische Tumordokumentation \\
\hline epidemiologisches Krebsregister \\
\hline klinisches Krebsregister \\
\hline Studien \\
\hline Qualitätssicherung \\
\hline interne Qualitätssicherung \\
\hline Abrechnung und DRG-Kodierung \\
\hline Vorgaben Lungenkrebszentrum (DKG, DGP, DGT) \\
\hline onkologische Zentren (DKG) \\
\hline Vorgaben Thoraxzentren (DGT) \\
\hline onkologische Zentren (DGHO) \\
\hline onkologische Vereinbarung (Kostenträger/KV) \\
\hline ambulante Behandlung im Krankenhaus nach §116b SGB V \\
\hline Qualitätssicherung ambulante Leistungen \\
\hline Qualitätsindikatoren S3-Leitlinie \\
\hline
\end{tabular}

Tab. 3 Module des einheitlichen onkologischen Basisdatensatzes [30].

Diagnosedaten
Operative Therapie
Strahlentherapie
Systemische Therapie
Verlaufsdaten
Abschlussdaten
Autopsiedaten

tronischen Tumordokumentationssystemen entsprechend strukturiert erfasst werden [30].

Für das Lungenkarzinom wurde im November 2011 zusätzlich der organspezifische Datensatz Lunge verabschiedet, der mit dem EGFR-Mutationsstatus nur einen einzigen weiteren evidenzbasierten Parameter enthält [30]. Die Behandlungskette für Patienten mit Lungenkarzinom, schematisch dargestellt in Abb.2, lässt sich mithilfe des einheitlichen onkologischen Basisdatensatzes bzw. des organspezifischen Datensatzes Lungenkarzinom bereits gut im Rahmen der klinischen Tumordokumentation für regionale bzw. nationale Vergleiche abbilden, da die wesentlichen prognosebestimmenden bzw. generell relevanten Dokumentationsparameter enthalten sind ( $\bullet$ Tab.4).

\section{Follow-Up-Erfassung}

Die Erfassung des Vitalstatus von erfassten Tumorpatienten ist essenziell für Analysen des Überlebens, dem letztendlich aussagekräftigsten Outcome-Parameter beim Lungenkarzinom. Aller-

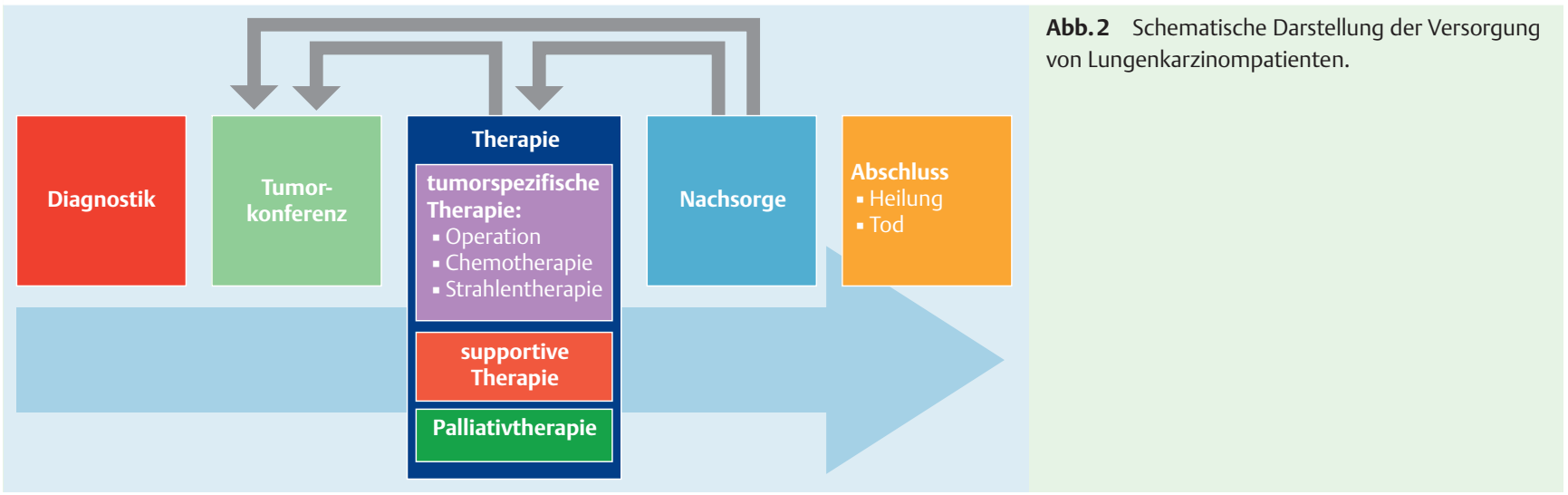


Tab. 4 Wesentliche prognosebestimmende bzw. generell relevante Dokumentationsparameter beim Lungenkarzinom.

\begin{tabular}{|l|}
\hline Diagnostik \\
\hline Histologie \\
\hline Molekularpathologie \\
\hline Staging \\
\hline Tumorkonferenz \\
\hline Therapieintention \\
\hline Konformität der Therapieentscheidung mit S3-Leitlinienempfehlung \\
\hline Therapie \\
\hline Operative Therapie \\
\hline Systemische Therapie \\
\hline Strahlentherapie \\
\hline Nachsorge \\
\hline Überlebensdaten \\
\hline Abschlussdaten \\
\hline
\end{tabular}

dings wird die regelmäßige Erhebung der Überlebensdaten durch die heterogene Landeskrebsregister- bzw. Landesdatenschutzgesetzgebung in einzelnen Bundesländern erschwert. Die prinzipiellen Möglichkeiten der Bestimmung des Vitalstatus aus der umfassenden Übersicht von Gumpp vom Klinischen Krebsregister des Tumorzentrums Freiburg gibt $\bullet$ Tab.5 wieder [31].

\section{Ausblick \\ $\nabla$}

Der Nationale Krebsplan enthält einen systematischen Aktionsplan zum Aufbau einer rationalen Tumordokumentation und Qualitätssicherung onkologischer Versorgung in Deutschland, basierend auf einer sorgfältigen Analyse und Problembeschreibung derzeitiger Tumordokumentationsstrukturen [5]. Die Kernprobleme uneinheitlicher gesetzlicher Regelungen, nicht aufeinander abgestimmter Dokumentationssysteme, einer Redundanz, fehlender Standardisierung und fragwürdiger Sinnhaftigkeit zu erfassender Parameter, des Datenschutzes, unzureichender zentraler und dezentraler Strukturen sowie der Finanzierung der Dokumentationsleistungen finden sich auch bei der Erfassung der Krankheitsdaten von Patienten mit Lungenkarzinom wieder. Die skizzierten Handlungsempfehlungen lassen sich gleichermaßen anwenden. Der einheitliche onkologische Basisdatensatz ermöglicht zusammen mit dem organspezifischen Datensatz Lungenkarzinom prinzipiell eine hinreichende Abbildung der Behandlung von Patienten mit Lungenkarzinom, in der Praxis findet er jedoch aufgrund der genannten Probleme keine flächendeckende Anwendung.

Speziell beim Lungenkarzinom sollten zusätzlich standardisierte Parameter mit entsprechender Methodik zur zukünftigen Erfassung von Lebensqualität, sozioökonomischen Daten, Komorbiditäten und spezifischen Bereichen der Palliativversorgung erarbeitet werden, da diese wesentlichen Aspekte der Patientenversorgung bislang nicht bzw. nur unzureichend durch die bisherigen Erfassungssysteme abgebildet werden, jedoch evidenzbasierte prognostische Relevanz besitzen [32,33].

Die Autoren dieser Arbeit sind überzeugt, dass Patienten mit Lungenkarzinom gerade wegen der schlechten Prognose einer vollständigen leitliniengerechten Betreuung bedürfen, welche auch tumorspezifische Behandlungsmaßnahmen in den fortgeschrittenen Tumorstadien bzw. bei Rezidivsituationen einschließt [1]. Hieraus resultiert aber der eindeutige Handlungsauftrag an die klinische Tumordokumentation beim Lungenkarzinom, die Effekte der Umsetzung von Leitlinienempfehlungen auch unter
Tab. 5 Möglichkeiten zur Erhebung des Vitalstatus nach Gumpp, Klinisches Krebsregister des Tumorzentrums Freiburg [31].

Anfragen bei den ehemaligen Patienten bzw. deren Angehörigen

Anfragen bei weiterbehandelnden Einrichtungen oder niedergelassenen Ärzten

Durchsicht der Todesanzeigen und Standesamtveröffentlichungen

in der Presse oder im Internet

Bezug von Leichenschauscheinen bzw. -daten von den Gesundheits-

ämtern

Bezug von Leichenschauscheindaten von den Epidemiologischen

Krebsregistern

Personenbezogene Anfragen bei Einwohnermeldeämtern per Brief oder per Internet

Elektronische Anfragen bei Einwohnermeldeämtern oder kommunalen

Rechenzentren per Datei

Pauschale elektronische Übermittlung von Sterbedaten oder Wegzugsdaten an Krebsregister

Bezug von Sterbedaten von der Deutschen Post AG

Bezug von Vitalstatusdaten aus Krankenhausinformationssystemen

Bezug von Vitalstatusdaten aus sonstigen behandlungsbegleitenden

Systemen

Recherchen in Internet-Telefonauskunftsseiten oder Telefon-

verzeichnissen auf Datenträgern

Einschluss einer Risikoadjustierung durch Hinzunahme der o.g. Faktoren wie z.B. Komorbiditäten zu untersuchen. Neben Überlebenszeitanalysen sollte hierbei auch die bislang vernachlässigte Betrachtung der Lebensqualität in den Mittelpunkt rücken.

Der generelle langfristige Nutzen von Tumordokumentation und Qualitätssicherung im Hinblick auf die Prognose von Lungenkrebspatienten wurde bislang noch nicht untersucht. Unseres Erachtens bedarf es aber unbedingt der Klärung dieser elementaren Frage nach der Legitimation ressourcenintensiver Dokumentationssysteme.

Im europäischen Vergleich rangiert Deutschland mit seiner heterogenen Tumordokumentation und Qualitätssicherung nur im Mittelfeld. Vorbilder können die gut aufeinander abgestimmten Systeme in Großbritannien [34] und Dänemark [35] sein, die beide eine ressourcensparende epidemiologische und klinische Tumordokumentation beim Lungenkarzinom unter Einbeziehung eines intelligenten Datenmanagements integrieren.

\section{Zusammenfassung \\ $\nabla$}

Angesichts der hohen Sterblichkeit und allenfalls mittelfristig zu erwartender messbarer Verbesserungen in der Therapie profitieren Lungenkrebspatienten am ehesten von einer flächendeckenden Implementierung der S3-Leitlinie und einer konsequenten Umsetzung der darin enthaltenen Behandlungsempfehlungen. Diese Hypothese gilt es, wissenschaftlich zu belegen, z.B. durch longitudinale Messung der Qualitätsindikatoren der S3-Leitlinie in Verbindung mit den Krebsregisterdaten. Es liegt jetzt an allen Akteuren, im Rahmen der zweiten Stufe des Nationalen Krebsplans Tumordokumentation und Qualitätssicherung konsequent zu entwickeln. Bei der konkreten Umsetzung muss die Versorgung von Lungenkarzinompatienten eine zentrale Rolle spielen.

\section{Interessenkonflikt \\ $\nabla$}

Die Autoren geben an, dass kein Interessenkonflikt besteht. 


\section{Literatur}

1 Goeckenjan G, Sitter H, Thomas $M$ et al. Prävention, Diagnostik, Therapie und Nachsorge des Lungenkarzinoms. Pneumologie 2010; 64 (Suppl. 02): e1-e164

2 Blum T, Schönfeld N, Kollmeier J et al. Lungenkrebs in Deutschland - zur Versorgungslage der Nation. Pneumologie 2011; 65: 7-18

3 Husmann G, Kaatsch P, Katalinic A et al. Krebs in Deutschland 2005/ 2006. Häufigkeiten und Trends. Robert Koch Institut und Gesellschaft der epidemiologischen Krebsregister in Deutschland e.V., Hrsg. 7. Ausgabe, Berlin: 2010

4 Ollenschläger G, Kirchner H, Fiene M. Leitlinien in der Medizin - scheitern sie an der praktischen Umsetzung? Internist (Berl) 2001; 42: $473-474$

5 Bundesministerium für Gesundheit. Nationaler Krebsplan vom 13.12.2011. http://www.bmg.bund.de/praevention/nationaler-krebsplan.html (abgerufen am 04.02.2012)

6 Bundesministerium für Gesundheit. Aktueller Stand des Nationalen Krebsplans (Umsetzungsempfehlungen) vom 04.01.2012. http:// www.bmg.bund.de/fileadmin/dateien/Downloads/N/Nationaler_Krebsplan/Informationspapier_Umsetzungsempfehlungen_NationalerKrebsplan_120104.pdf (abgerufen am 04.02.2012)

7 Sens B, Fischer B, Bastek A et al. Begriffe und Konzepte des Qualitätsmanagements. GMS Med Inform Biom Epidemiol 2007; 3: Doc05

8 Blum T. Aktuelle Versorgungslage von Patienten mit Lungenkrebs in Deutschland und Aufbau eines offenen Qualitätsmanagement-Systems zur kontinuierlichen Verbesserung der Versorgungsqualität bei Lungenkrebs. [Masterarbeit] Lüneburg: Universität Lüneburg; 2008

9 Donabedian A. Evaluating the quality of medical care. Milbank Memorial Fund Quarterly 1966; 44: 166-203

10 Ruprecht $T$. Von der Qualitätssicherung zum Qualitätsmanagement. Entwicklung in der vertragsärztlichen Versorgung. Z Allgemeinmedizin 1993; 69: Epub 967

11 Deutsches Institut für Normung (Hrsg.). Qualitätsmanagementsysteme - Grundlagen und Begriffe (ISO 9000:2005). Dreisprachige Fassung EN ISO 9000:2005. Berlin: Beuth; 2005

12 Ministerium für Justiz, Gesundheit und Soziales. 40 Jahre epidemiologisches Krebsregister Saarland vom 01.06.2007. http://www.krebsregister.saarland.de/publikationen/PDF/Publikation40Jahre.pdf (abgerufen am 04.02.2012)

13 Hentschel S, Pritzkuleit R, Schmid-Höpfner S et al. Epidemiologische Krebsregistrierung in Deutschland - Aufgaben und aktueller Status. Onkologe 2011; 17: 97-106

14 Altmann $U$, Hofstädter H, Katalinic A et al. Klinische und Epidemiologische Krebsregister in Deutschland. Forum DKG 2009; 24: 19-22

15 Gesetz über Krebsregister (KRG). Bundesgesetzblatt Teil I 1994; 79: $3351-3355$

16 Bundeskrebsregisterdatengesetz (BKRG). Bundesgesetzblatt Teil I 2009; 53: 2707-2708

17 Schön D, Bertz J, Görsch B et al. Die Dachdokumentation Krebs - Eine Surveillance-Einrichtung der Krebsregistrierung in Deutschland. Bundesgesundheitsbl Gesundheitsforsch Gesundheitsschutz 2004; 47: 429-436

18 Wolf $U$, Barnes B, Bertz $J$ et al. Das Zentrum für Krebsregisterdaten (ZfKD) im Robert Koch-Institut (RKI) in Berlin. Bundesgesundheitsbl Gesundheitsforsch Gesundheitsschutz 2011; 54: 1229-1234

19 Kooperationsverbund Qualitätssicherung durch klinische Krebsregister. Klinische Krebsregister vom 13.01.2012. http://www.koqk.de/klinische-krebsregister.html (abgerufen am 04.02.2012)

20 Hofstädter F, Klinkhammer-Schalke M. Aufgaben und Strukturen für die klinische Krebsregistrierung. Onkologe 2011; 17: 121-125
21 Arbeitsgemeinschaft Deutscher Tumorzentren AD. Zur Konzeption und zum Personal- und Finanzbedarf der Tumorzentren in Deutschland - Memorandum der Arbeitsgemeinschaft Deutscher Tumorzentren e.V. (ADT) vom 17.03.2006. 5. neu bearbeitete Auflage. http://www.tumorzentren.de/tl_files/dokumente/ADT.Memorandum. letzte.Fassung.pdf (abgerufen am 04.02.2012)

22 Schönfeld N. Persönliche Kommunikation vom 05. 022012

23 Deutsche Gesellschaft für Thoraxchirurgie. Anforderungskatalog an ein Thoraxzentrum - Kompetenzzentrum für Thoraxchirurgie - der Deutschen Gesellschaft für Thoraxchirurgie vom 08.11.2008. http:// www.dgt-online.de/fileadmin/Daten/pdf/Anforderungskatalog_Thoraxzentrum_2008-11-08.pdf (abgerufen am 04.02.2012)

24 Deutsche Krebsgesellschaft. Erhebungsbogen für Lungenkrebszentren der Deutschen Krebsgesellschaft vom 18.04.2011. http://www.onkozert.de/lungenkrebszentren.htm (abgerufen am 04.02.2012)

25 Kassenärztliche Bundesvereinigung. Vereinbarung über die qualifizierte ambulante Versorgung krebskranker Patienten „Onkologie-Vereinbarung" (Anlage $7 \mathrm{zu}$ den Bundesmantelverträgen) vom 31.10.2011. http://www.kbv.de/rechtsquellen/2297.html (abgerufen am 04.02.2012)

26 Deutsche Gesellschaft für Hämatologie und Onkologie. Grundsatzpapier „Onkologische Zentren“ Version 2.4 vom 31.3.2010. http://www. dgho.de/zertifizierungen/onkologische-zentren/antragsformulare (abgerufen am 04.02.2012)

27 Merk J, Jagota A, Schicke B et al. Können klinische Krebsregister einen Beitrag zur Evaluation der Versorgungsqualität des Lungenkarzinoms leisten? Atemw-Lungenkrkh 2010; 36: 114-119

28 Blatt $K$, Hölterhoff $M$, Henkel $M$ et al. Aufwand-Nutzen-Abschätzung zum Ausbau und Betrieb bundesweit flächendeckender klinischer Krebsregister vom 15.10.2010. http://www.bundesgesundheitsministerium.de/fileadmin/redaktion/pdf_publikationen/Gutachten-Aufwand-Nutzen-Abschaetzung-Krebsregister.pdf (abgerufen am 04.02. 2012)

29 Querschnitts-AG Dokumentation. Datensparsame einheitliche Tumordokumentation - eine Kernforderung des Nationalen Krebsplans vom 10.10.2011. http://www.bundesgesundheitsministerium.de/filead$\mathrm{min} /$ dateien/Downloads/N/Nationaler_Krebsplan/Papier_zur_Tumordokumentation_101011.pdf (abgerufen am 04.02.2012)

30 Arbeitsgemeinschaft Deutscher Tumorzentren, Gesellschaft der epidemiologischen Krebsregister in Deutschland. Gemeinsamer einheitlicher Onkologischer Basisdatensatz von 08/2008. http://www.tumorzentren.de/onkol-basisdatensatz.html (abgerufen am 04.02.2012)

31 Gumpp V. Vitalstatusermittlung vom 02.07.2010. http://www.uniklinik-freiburg.de/tumorzentrum/live/Wir-ueber-uns/ Klin-Krebsregister/follow-up/vitalstatusermittlung.html? raw=true\&layout=weiss\&szsrc= (abgerufen am 04.02.2012)

32 Dalton SO, Frederiksen BL, Jacobsen E et al. Socioeconomic position, stage of lung cancer and time between referral and diagnosis in Denmark, 2001-2008. Br J Cancer 2011; 105: 1042 - 1048

33 Rich AL, Tata LJ, Free CM et al. Inequalities in outcomes for non-small cell lung cancer: the influence of clinical characteristics and features of the local lung cancer service. Thorax 2011; 66: 1078-1084

34 Rich AL, Tata LJ, Stanley RA et al. Lung cancer in England: information from the National Lung Cancer Audit (LUCADA). Lung Cancer 2011; 72: $16-22$

35 Jakobsen E, Palshof T, Osterlind $K$ et al. Data from a national lung cancer registry contributes to improve outcome and quality of surgery: Danish results. Eur J Cardiothorac Surg 2009; 35: 348-352 